\title{
UNA REVISION CRÍTICA DE INVESTIGACIONES HECHAS EN COLOMBIA SOBRE EDUCACION Y ECONOMIA*
}

Bernardo Kugler Wagenberg**

El objetivo de este documento consiste en examinar trabajos de investigación referidos a Colombia, que tocan de alguna manera la relación entre procesos económicos y educativos. La referencia a Colombia debe ser específica, así sólo se mencione un mínimo necesario de trabajos teóricos generales sin aplicación concreta al caso colombiano.

Para delimitar los trabajos considerados sería también necesario definir los conceptos de economía y educación; obsérvese sin embargo, que de ellos pueden hacerse definiciones bastante restrictivas o casi tan amplias como se quiera, así, en lugar de entrar en una profunda discusión al respecto, se especifica que en general el término educación se refiere principalmente a aquella que es escolarizada o formal" y para la cuestión de la economía los límites se imponen por el lado de una definición disciplinaria ${ }^{54}$; por ninguno de los dos conceptos se va a ser totalmente excluyente, aunque sí se espera una muy amplia inclusión de los trabajos hechos que caen dentro de la definición.

A continuación, en el numeral I se tratan trabajos que consideran aspectos económicos del sistema educativo, principalmente los de costos y financiación, extendiéndose a problemas de eficiencia interna y a factores determinantes de la educación y la escolaridad relacionados con financiación, pero excluyendo el enfoque del capital humano.

El numeral II contiene estudios de capital humano y de requerimientos de recursos humanos, así como aquellos relacionados a líneas de causalidad de la educación hacia componentes socioeconómicos tales como los mercados de trabajo.

Finalmente en el numeral III se discuten investigaciones que han tratado de alguna manera la relación estructural de la educación y la escolaridad con el sistema económico; en general, resultados parciales de ellas ya habrán sido mencionados en los numerales anteriores y aquí se discutirá el problema de las relaciones como tales.

En el procedimiento seguido en este trabajo para cada tema, se hace primero un breve sumario de los principales resultados de cada investigación, comentándose luego la posible validez de los mismos, para finalmente tratar de identificar el "estado de las artes" y sugerir posibles líneas futuras de trabajo.

\footnotetext{
* Trabajo presentado al Seminario Nacional de Investigación Educativa, organizado por el Instituto SER de Investigación y patrocinado por el Fondo Colombiano de Investigaciones Científicas "Francisco José de Caldas" (COLCIENCIAS).

${ }^{* *}$ Director Área Socio-Económica CCRP. Asesor del estudio recursos humanos SENA-Holanda. Ingeniero Civil. U. Nacional M.A. y candidato a Ph. D. (en Economía) en la U. de Minessota.

${ }_{54}$ Para aclaraciones en estos aspectos, remitirse al documento de Vielle circulado por los organizadores del Seminario.
} 


\section{Aspectos Económicos del Sistema Educativo}

Los problemas de financiación, costos, calidad y eficiencia ${ }^{55}$ de los programas de educación están altamente relacionados entre sí. Efectivamente analizar costos independientemente de la calidad y la eficiencia, puede llevar a conclusiones tendenciosas y a circunstancias especiales alrededor de costos; calidad y eficiencia pueden conducir a justificar cierto tipo de financiación. Así por ejemplo, un programa que presenta mayores costos <unitarios) puede ser ineficiente si su calidad no es superior; en tanto que puede justificarse si es eficiente y su mayor calidad compensa, de acuerdo con ciertas normas, los mayores gastos. Circunstancias variantes en estos aspectos pueden a su vez justificar financiación especial para ciertos programas.

El objetivo último de la investigación de la economía del sistema educativo debe ser la identificación de programas eficientes para satisfacer las necesidades sociales al respecto; aquí se plantea un problema de tipo técnico para poder medir la eficiencia y un problema de tipo político para definir qué quiere decir satisfacer necesidades. Vale la pena observar que este tipo de planteamientos no son exclusivos a la cuestión del sistema educativo sino que se pueden generalizar como temas de la problemática económica. Teniendo en cuenta el presente enfoque se puede entrar a analizar en qué medida se ha avanzado en Colombia en la investigación de estos temas.

En cuanto a la financiación de la educación se refiere, fuera de la información que se genera de las encuestas de presupuestos familiares, nada más se sabe sobre los recursos privados que a ella se dedican. Sobre financiación pública se ha avanzado bastante desde los años sesenta, cuando todavía era bastante complicado concretar detalles al respecto ${ }^{56}$. En diversas publicaciones de las instituciones públicas correspondientes aparece información sobre las localizaciones presupuéstales y ejecuciones del sector discriminadas por niveles y por unidades geográficas; quedaría pendiente como información interesante la que permitiera discriminar las asignaciones para educación rural. Jallade y Selowsky (1979) han analizado el impacto distributivo del gasto público en educación y encuentran que es altamente progresivo para primaria y aún secundaria, pero es aproximadamente neutral o un poco regresivo para educación superior; este último resultado es poco preciso por cuestión de tamaño muestral. Existen como cálculo intermedio en los mencionados trabajos datos aproximados de costos escolares.

A partir del gasto público y su eficiencia, Kugler y Vivas estudiaron la posible evolución de la cobertura del sistema escolar colombiano y encontraron que ella llegaría pronto a altos valores en el sector urbano, pero que en la zona rural se mantendrían vigentes los problemas hasta finales del siglo.

Los resultados anteriores deben relacionarse con la cuestión de costos. Aun cuando se ha avanzado en ello y se han identificado algunas escalas adecuadas de operación en los estudios de Jallade y Reyes y Restrepo, tales estudios usan principalmente datos de sección transversal, por lo cual no se tiene claridad sobre lo que ha pasado al respecto en el país a lo largo del tiempo. Jallade y Reyes para primaria y Restrepo para secundaria encuentran que las áreas más urbanizadas del país presentan menores costos unitarios

\footnotetext{
${ }^{55}$ Se usa el término eficiencia aquí, en el sentido económico de utilizar un mínimo de recursos para lograr ciertos objetivos; estos objetivos no deben ser la educación per-se, sino que deben incluir una función socioeconómica más amplia.

${ }^{56}$ Se arguía en general que era imposible evitar la doble contabilidad de ciertas partidas que aparecían como rubros del gobierno central y, además, después de transferidos como gastos de otras unidades administrativas.
} 
gracias a mejores escalas de operación. Las disparidades conceptuales y metodológicas entre estudios de épocas diferentes, donde se calculan costos, hacen imposibles sus comparaciones. El estudio de costos universitarios que propone el Instituto Colombiano para el Fomento de la Educación Superior (ICFES) y que ha empezado a ejecutarse es un avance en esta dirección.

Vale la pena llamar la atención sobre el hecho de que existen diferencias entre la concepción contable y la concepción económica de costos. Para el análisis económico interesa la segunda y según ella, no es necesario calcular en detalle valores depreciados de activos fijos sino evaluar el costo de oportunidad del uso de los mismos ${ }^{57}$, ni tampoco gastar muchas energías en metodologías para asignar los costos indirectos ${ }^{58}$.

Estas discusiones tienen que ver mucho con la eficiencia y la calidad del proceso educativo. Así, los costos unitarios por alumno pueden reducirse por aumentar el número de alumnos por maestro y ser esto un movimiento correcto al pasar, dígase, de diez a veinticinco alumnos por maestro, o ser un movimiento incorrecto por pasarse a aulas donde hay cincuenta alumnos por maestro. Similarmente, no se puede tachar apresuradamente de inadecuada (léase ineficiente) una institución donde los costos parecen excesivamente altos y ello resulta así porque los profesores tienen un alto nivel de preparación.

Resulta notoria la escasez de estudios que arrojen resultados más o menos sólidos en estos aspectos. Usualmente se encuentran algunas "evaluaciones" realizadas por la institución interesada, como por ejemplo el Ministerio de Educación Nacional o el Servicio Nacional de Aprendizaje (SENA), donde se consignan algunos indicadores cuestionables que muestran el éxito (o fracaso) del programa evaluado. En realidad, aun cuando hay limitaciones metodológicas, existe ya cierto consenso sobre la existencia de algunos indicadores de logros que pueden aplicarse para evaluar la eficiencia de ciertos programas educativos; los logros de cada programa deben contrastarse con los respectivos costos para tener ideas sobre su eficiencia relativa. Parece que no hay en Colombia un solo estudio que haga esto.

Existe sí, cierto tipo de evidencia "blanda" sobre qué puede haber estado sucediendo en el sector educativo. Así entonces, se presume que los altos aumentos en las asignaciones presupuéstales reales junto con las reducciones en la tasa de crecimiento de la población escolarizable han incrementado la cobertura y la promoción; parece haber habido reducciones en los niveles le deserción y repetición pero esto no ha podido comprobarse, por cuanto -las cifras de primaria y secundaria que oficialmente se conocen desde 1969 son de proyecciones; resulta aún más difícil atribuir a ciertas causas estas posibles mejoras del sistema, pero sí parece sugestivo pensar en que la calidad y actitud general de los maestros puede jugar algún papel en el proceso. Estos argumentos no muy apoyados en una realidad estadística pueden aparecer más o menos razonables, pero fácilmente se pueden volver más o menos irrazonables, y es así como se encontrarían afirmaciones indicando que hay demasiados normalistas en Colombia o que ciertos institutos técnicos son muy costosos porque la mayor parte de sus maestros son profesionales egresados de la universidad.

\footnotetext{
${ }^{57}$ Resulta más preciso con estos propósitos calcular el valor de arrendamiento por metro cuadrado de un local similar a una escuela y el número de metros cuadrados por estudiante, que llevar una contabilidad cuidadosa de costos de construcción y su depreciación.

${ }^{58}$ Es imposible discernir, con exactitud, los costos de administración central de una institución entre sus departamentos o entre sus grupos, así que cualquier metodología medianamente razonable sirve para asignar costos indirectos. 
Infortunadamente Colombia en estos aspectos se mueve más con base en los prejuicios políticos y sociales de turno, que con información fundamentada en investigaciones con cierta objetividad. Esto, en cierto grado, se debe al interés que las mismas instituciones patrocinadoras de las evaluaciones tienen sobre los aspectos evaluados; debe sin embargo, en la medida en que se desee una verdadera investigación aplicada, buscar un mínimo de autonomía por parte del investigador. Esto parece lograrse cuando el investigador se mueve hacia temas más generales.

Existen varios trabajos en los cuales aparece cómo los niveles educativos dependen de las condiciones de desarrollo. A nivel macro lo analizan Cataño, L3bot (1972), Parra (1973), Parra (1974), Parra (1978) y Mojica. Particularmente Lebot y Parra muestran cómo el desarrollo del sistema educativo colombiano responde a las condiciones y necesidades del desarrollo socio-económico, lo cual se hace más evidente a nivel regional. A nivel de familias, Kugler, Urrutia $(1974$, a) y Fields encuentran que la escolarización de las personas depende en buen grado de las condiciones socioeconómicas de sus familias paternas ${ }^{59}$. Un resultado particularmente interesante consiste en que el uso de un índice múltiple de condiciones socioeconómicas no adiciona gran cosa por encima de un índice simple bien seleccionado, tal como escolaridad de la madre.

Urrutia (1974, b) no utiliza datos sobre Colombia, pero espera que "...la democratización de la educación reduzca los diferenciales de ingresos”. Lo que aparece confirmado en el estudio de Jallade y con mayor fuerza en el de Selowsky (1979), quien analizando una muestra especialmente seleccionada con dicho propósito logró encontrar gran poder redistributívo en el gasto público en educación primaria y secundaria, pero no en el de educación superior; que no hacía parte de la muestra, pero podría esperarse que la democratización que ha comenzado para la primaria urbana, gradualmente llegue a los niveles superiores también.

\section{Los Efectos de la Educación}

Parece en la actualidad estar ampliamente aceptado de que existe un alto grado de asociación entre los niveles de escolaridad de las personas y sus niveles de ingreso. Este tipo de asociación se observó inicialmente para países sin que se haya podido dilucidar plenamente la discusión sobre si el nivel de desarrollo socioeconómico es causa del nivel educativo o viceversa; problema que será tratado en el numeral siguiente y por ahora, siguiendo a Blaug, se discuten los trabajos que de alguna manera presentan los "sorprendentes efectos de la educación".

Después de los esfuerzos pioneros de G. Franco y de A. Franco, aparecen en primera instancia los trabajos de Selowsky (1969) y Schultz que, por la misma época cuando el sistema escolar colombiano empezaba a expandirse notablemente, muestran las altas tasas de rentabilidad de la escolaridad primaria y secundaria, tanto a nivel privado como social. Contrasta con este resultado el de la tasa de rentabilidad social de la educación superior que ambos autores encuentran en un $5 \%$ anual. Al revisar el resultado muestra

\footnotetext{
${ }^{59}$ Curiosamente Fields afirma que el sistema educativo es abierto (democrático) construyendo un índice que lo muestra. Se puede construir otro índice que, con los mismos datos, muestre lo contrario. En general cualquier índice es arbitrario y es mejor no jugar con ellos. En este caso, todos los investigadores encontraron un coeficiente de determinación de cerca del $50 \%$ entre escolaridad y antecedentes socioeconómicos, lo que muestra una relación bastante estrecha. 
un error de cálculo en Schultz y una imputación adicional de costos en Selowsky, las cuales en caso de corregirse pueden llevar la tasa a cerca de un $10 \%$ anual ${ }^{60}$.

El trabajo posterior de Dougherty encuentra similarmente altas rentabilidades para primaria y secundaria y una del $7.5 \%$ anual para la superior. Con base en estos resultados Dougherty encuentra la forma óptima como debe crecer la cobertura del sistema educativo en Colombia, buscándose a la mayor brevedad posible la cobertura total de primaria, creciendo al $15 \%$ anual la secundaria hasta 1980 y manteniendo luego la cobertura de los egresados de primaría, en tanto que la educación superior sólo debe crecer al $2 \%$ anual hasta 1980 y luego al $5 \%$ anual.

Los resultados anteriores merecen algunos comentarios adicionales. En primer lugar, los tres trabajos coinciden bastante en presentar alrededor del $20 \%$ anual como tasa de rendimiento de la secundaria. La de primaria en cambio difiere en ellos, aun cuando se mantiene alta; la razón para ello está en la dificultad de calcular con precisión los costos de oportunidad de jóvenes en edad de asistir a primaria, los cuales son cercanos a cero ${ }^{61}$. Por otro lado, llama, la atención los bajos valores de la rentabilidad a la educación superior, resultado que junto con los mencionados sobre el carácter de mala redistribución del gasto público en la misma deben llevar a la conclusión que el Gobierno debía haber detenido el gasto, en los programas correspondientes. Solamente cinco años después en los trabajos de Kugler (1975) y de Kugler, Reyes y Gómez aparecen valores por encima del $10 \%$ anual para la rentabilidad (privada) de la educación superior. Aún puede argumentarse que la rentabilidad privada excede a la social, sin embargo el mejor indicio sobre si la educación superior es rentable o no, a falta de mejor evidencia, puede darlo el crecimiento de la matrícula en universidades privadas donde los costos son plenamente cubiertos por los estudiantes. Quizá algunos estudios sobre rentabilidad por tipos de carreras puedan arrojar nuevas evidencias sobre el tema.

Otro aspecto interesante en los trabajos de Selowsky (1969) y Dougherty está en que, cuando entran a considerar explícitamente sustitución entre tipos de trabajo, deben suponer una tasa de sustitución muy alta ${ }^{62}$ para poder mantener tasas de rentabilidad adecuadas. Esto apunta hacia un supuesto importante que usualmente se hace en estudios de capital humano y que puede cuestionarse: la demanda por los tipos de trabajo será tal que el nivel de empleo se mantendrá. El estudio de Kugler y Vivas que se basa en el supuesto contrario de coeficientes fijos, apunta en una dirección similar, en el sentido que muestra cómo los requerimientos educativos de la demanda por trabajo quedaban ampliamente satisfechos desde 1975.

Sucede que el problema de los efectos de la educación sobre el aparato productivo no es el resultado de la oferta, como lo supone inicialmente el enfoque del capital humano, o de la demanda como lo supone el enfoque de recursos humanos. El primero lleva sembrado en sí el resultado de educar más, el segundo el de no educar más. Actualmente están prácticamente erradicados los estudios con esta clase de preconcepciones y más bien se enfocan en la dirección de ver la importancia relativa que la oferta y la demanda, de los diferentes tipos de trabajo, tienen en la determinación del funcionamiento de los mercados de trabajo.

\footnotetext{
${ }^{60}$ Selowsky utiliza cifras de la Universidad Nacional para el cálculo de costos. La inversión en esa época estuvo bastante por encima de los promedios y además Selowsky incluye todo el presupuesto de Bienestar Estudiantil, la mayor parte del cual <cafetería, residencias, servicio médico> claramente no es costo educativo.

${ }^{61}$ Con un costo de oportunidad de cero, la rentabilidad calculada puede pasar del $100 \%$ anual y con un valor cercano al salario mínimo se obtiene alrededor de un $20 \%$ anual.

${ }^{62}$ En estudios econométricos es raro encontrar tasas de sustitución entre factores productivos muy por encima de la unidad. Selowsky usa tres y Dougherty aun mayores valores. 
En estas líneas el principal cuestionamiento va en la dirección de la medida en que el salario se relaciona con la productividad en cuanto se acepten las definiciones convencionales de productividad en economía, se puede mostrar la estrecha relación de ésta con los salarios ${ }^{63}$. Otros cuestionamientos respecto a la posibilidad que la rentabilidad de la educación refleje efectos de cambios en productividad se plantean en términos de lo que Blaug denomina las hipótesis "sociológica" y la "sicológica". Según la primera, la educación solamente genera mayores ingresos en cuanto refleja diferencias de clase social y para la segunda la educación es sólo una credencial para ocupar los puestos mejor remunerados.

Tal como se mencionó en el numeral anterior, existe evidencia amplia sobre cómo en Colombia la composición educacional de la población resulta de una cierta estructura de clases, sin embargo, algunos de esos mismos estudios muestran ${ }^{64}$, de una manera más 0 menos concluyente, cómo los efectos de la escolaridad se mantienen, aun al controlar los antecedentes socioeconómicos, para la fuerza de trabajo urbana.

La hipótesis del credencialismo se puede rechazar a nivel de comportamiento individual de los empresarios, por cuanto si la mayor escolaridad no reflejara una mayor productividad, los empresarios no estarían mostrando un comportamiento de maximización de ganancias. Sin embargo, a nivel 'macro" se puede presentar una sugestiva teoría de estratificación social, como aparece en el trabajo de Corchuelo, donde se argumenta que, en presencia de un ejército de reserva de trabajo, se utiliza la estructura educativa como medio para mantener las expectativas y el orden existente en los mercados de trabajo. Corchuelo presenta cifras de mercados de trabajo en el Valle del Cauca coherentes con los postulados mencionados. Nuevamente aquí, debe arguírse que para mantenerse un comportamiento agregado de este tipo, los empresarios deben obtener beneficios de corto plazo reflejados de alguna manera por cambios en productividad $^{65}$.

Aceptándose que en general, los estudios con enfoque de capital humano que presentan altas rentabilidades de la educación muestran algún efecto productivo de ésta, se justifica mencionar que Puryear y Maldonado encuentran resultados muy positivos en la misma dirección para el SENA. En todo caso se considera prudente examinar los resultados de rentabilidad con el fin de identificar razones específicas a las cuales se pueden deber. Así Puryear analiza movilidad ocupacional de egresados SENA y encuentra que buena parte de las altas rentabilidades se puede deber a esa movilidad.

Similarmente, Kugler, Reyes y Gómez encuentran que las rentabilidades a la escolaridad no difieren estadísticamente entre sectores modernos y no modernos (urbanos) y que esto se debe en buena medida a una alta movilidad intersectorial generada por la alta tasa de crecimiento de la fuerza de trabajo ${ }^{66}$.

Reyes y Gómez encuentran en su trabajo que, desde el punto de vista de rentabilidad, la educación técnica no presenta diferencias con respecto a la clásica, pero analizando

\footnotetext{
${ }^{63}$ Véase, por ejemplo, Kugler, Reyes, Gómez para una discusión al respecto.

${ }^{64}$ Particularmente los de Kugler (1974), Urrutia (1974b) y Fields.

${ }^{65}$ Solamente el sector público puede mantener un comportamiento diferente. Este sector ha sido el principal generador de nuevos empleos en el país desde hace cerca de treinta años; el trabajo de Kugler, Reyes y Gómez sugiere que el sector público actúa como seguidor del sector privado en materia de determinación de salarios y en este sentido podría estar actuando como credencialista, sin embargo, parece razonable suponer que si la educación tiene efectos productivos en el sector privado, también debe tenerlos en el sector público.

${ }^{66}$ En una fuerza de trabajo que crece a un $5 \%$ anual, puede darse una relocalización interna sustancial de ella en un período de 5610 años, sin que los trabajadores antiguos se muevan.
} 
composición social del enrolamiento, pruebas académicas e inserción en la fuerza de trabajo, resulta que la educación técnica puede ser un instrumento de mejoramiento educativo para estratos sociales bajos.

Haller tiene el único trabajo sobre rentabilidad de la educación rural, basada en producción agropecuaria. Inicialmente encuentra que no hay efectos directos significativos de la escolaridad sobre el ingreso, pero refinando el enfoque y teniendo en cuenta producción, halla efectos importantes sólo cuando se utilizan tecnologías modernas. Este resultado coincide con la apreciación de Lebot sobre el carácter no rural que históricamente ha tenido la educación formal rural en Colombia. Parra (1978) sugiere algo similar en su estudio sobre zonas cafeteras, sin embargo no existe en el país evidencia concluyente sobre el carácter "expulsor" que pudiera tener la educación rural.

\section{La Interacción Educación-Economía}

La dicotomía hecha entre los dos numerales anteriores es, en cierto grado, poco rigurosa ya que los factores de decisiones públicas y privadas que inciden en la determinación de la educación dependen de los efectos esperados que ésta puede tener, y a su vez los resultados de la educación dependen de los determinantes de la misma, no solo indirecta sino también directamente.

Algunos de los estudios ya mencionados, en particular los de Lebot, Parra y Kugler, postulan y muestran como en general el problema de los efectos de la educación no es independiente de todo el entorno económico. Es por lo tanto necesario en esta clase de estudios tener un panorama claro sobre la relación general que, en ambas direcciones, se da entre educación y economía, lo cual no quiere decir que cada estudio debe seguir un enfoque totalizante incluyendo todas las relaciones posibles. Precisamente una de las funciones de la investigación consiste en poder aislar ciertos efectos importantes.

Parra en sus diferentes trabajos, utilizando análisis de tipo regional muestra de una manera convincente que los niveles de desarrollo socioeconómico determinan la estructura educacional, pero debe llamarse la atención sobre como ésto no permite concluir que "La educación como fuente de movilidad social general es un mito. La educación como clave del desarrollo y más aún como condición suficiente y necesaria

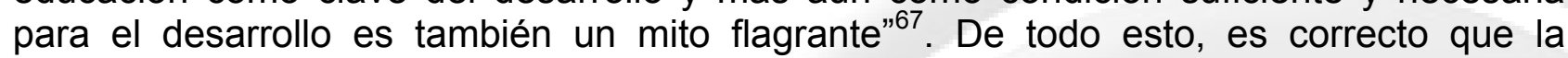
educación no es condición suficiente para el desarrollo, pero precisamente en este trabajo y en otros Parra muestra que la educación sí genera movilidad, al menos espacial, y que el hecho de concentrarse la educación en las regiones de mayor desarrollo apunta más bien hacia la educación como condición necesaria para el desarrollo socioeconómico.

Mojica en sus trabajos enuncia la inutilidad de la educación como instrumento de desarrollo o de generación de ingresos, para ello se remite a trabajos escritos con referencia a los Estados Unidos y traslada sus implicaciones al caso colombiano sin preocuparse por los resultados aquí obtenidos ${ }^{68}$.

El ya mencionado trabajo de Corchuelo postula otros dos aspectos que vale la pena discutir. Uno es la desigualdad de ingresos que se presenta aun a iguales niveles educativos; lo cual es un hecho, efectivamente los modelos de capital humano explican

\footnotetext{
${ }^{67}$ Traducido de Parra (1973) p. 77.

${ }^{68}$ Uno de los trabajos en que Mojica basa sus resultados es en el de Christopher Jenks [Inequality. Basic Books, New York, 1972]. Este voluminoso libro es un ejemplo de como hasta un profesor de Harvard se puede equivocar, pues todos los resultados se basan en la incorrecta aplicación de técnicas estadísticas.
} 
entre un $25 \%$ y un $50 \%$ de la desigualdad de ingresos del trabajo, es decir, faltaría aún por explicar más de la mitad de la desigualdad y sin tener en cuenta todavía la desigualdad de ingresos totales generada por la distribución de la riqueza; debe ser claro que los modelos de capital humano no explican mucho de la desigualdad de ingresos, pero son los que, dentro de un contexto teórico, son más claros. Falta mucho por explicar y las alternativas de antecedentes socioeconómicos o de segmentación de mercados explican estadísticamente menos por sí solas, y muy poco, en relación con la educación, en cuanto a la distribución del ingreso. Esto se hace evidente en los trabajos de Kugler (1974), Reyes y Gómez.

El otro tema de discusión que se plantea a partir de la investigación de Corchuelo es el de la "improductividad" del empleo en sectores terciarios. Cierto que resulta inconcebible una sociedad produciendo solamente servicios, pero actualmente se reconoce la necesidad del funcionamiento de ciertos sectores de servicio para promover un desarrollo económico. Para ganar claridad en estos temas resulta necesario estudiar el papel que los sectores de la producción deben jugar en el proceso de desarrollo económico y, a su vez, el papel que la educación juega en el desarrollo de la respectiva fuerza de trabajo.

Esto requiere análisis de tipo dinámico, de los cuales mas bien pocos se han hecho en Colombia, debido quizá en buena parte a la escasez de datos de series de tiempo suficientemente largas ${ }^{69}$. Sólo recientemente se empieza a contar con datos como para analizar los cambios que aparentemente se han presentado en el papel de los diferentes niveles educativos en la economía, tal como parecen sugerirlo los cambios en las tasas de rentabilidad por niveles educativos y los cambios en la composición educativa del desempleo.

La forma como han interactuado el sistema educativo formal y los sectores productivos en la parte urbana parece indicar que la educación formal general cumple un papel adecuado de acuerdo con el proceso de desarrollo económico urbano, en el cual las mayores posibilidades de movilidad resultan más una virtud que un defecto.

Estudios específicos de programas educativos deberán explorar esta hipótesis, enmarcándose dentro de la concepción de las relaciones generales entre educación y economía. Ni el papel de la educación rural, ni sus determinantes aparecen claros en el momento. Los estudios del Ministerio de Educación Nacional (1976 y 1978) parecen proporcionar un punto de partida en este campo, donde los conocimientos son tan precarios que no se sabe si la educación rural educa para el campo o para la ciudad, ni a qué se deben sus bajos niveles de eficiencia.

Este trabajo ha reseñado cerca de medio centenar de estudios. No es una cantidad excesiva, pero tampoco despreciable. Ha habido aportes importantes, con un grado de generalidad amplio, que indican la importancia de la educación en el proceso socioeconómico. Debe entrarse ahora en la investigación de la dinámica de estas relaciones y de cuestiones específicas, lo cual eventualmente permita la identificación de mejores instrumentos para influenciar positivamente el proceso de desarrollo.

\footnotetext{
${ }^{69}$ Obsérvese que un ciclo educativo completo lleva cerca de quince años; para analizar transiciones en el mismo se requerirían datos siquiera de treinta años.
} 


\section{BIBLIOGRAFÍA}

ALZATE, José y Rodrigo Parra. Los Determinantes de la Escolaridad. "Aportes para el Análisis de la Educación Urbana en Colombia". Universidad de los Andes. Bogotá. 1976.

BLAUG, M. La Educación y el Problema del Empleo en los Países en Desarrollo. OIT. Ginebra, 1973.

CATAÑO, Gonzalo. Universidad Pública y Movilidad Social. Introducción al Estudio de la Educación y la Movilidad Social en Colombia. Bogotá. 1969.

CATAÑO. Gonzalo. "Escolaridad y Movilidad Social". Boletín Mensual de Estadística [DANE]: No. 243, pp. 175-191. Bogotá, 1971.

CORCHUELO. Alberto. Educación y Asignación de la Fuerza de Trabajo: El Caso del Valle del Cauca. Universidad del Valle. CIDSE. Cali. 1978.

CORPORACION CENTRO REGIONAL DE POBLACION. Modelo SERES (Sistema para el Estudio de las Relaciones Económicas, Sociales y Demográficas). "Descripción Técnica". CCRP. Área Socio-Económica. Bogotá. 1975.

DOUGHERTY, Christopher. "El Futuro de la Educación Colombiana: Proyecciones y Prioridades”. Revista de Planeación y Desarrollo. III. 1. 1971. Bogotá.

FALS, Orlando. La Educación en Colombia: Bases para una interpretación Sociológica. Universidad Nacional. Bogotá. 1967.

FIELDS. Gary. Educación y Movilidad Económica en Colombia. Universidad de los Andes. Bogotá. 1977.

FRANCO. Augusto. Investment on Education (Colombia and USA). (mimeo).

FRANCO, Guillermo. Rendimiento de las inversiones en Educación en Colombia. Universidad de los Andes. Bogotá. 1964.

GOMEZ, Víctor M. Credencialismo, Dualismo Laboral y Desarrollo Educativo. Universidad del Valle. Cali. 1977.

HALLER, Tomas. Education and Rural Development in Colombia. Michigan Xerox, 1972. Tesis Purdue University, Lafayette.

ICFES. División de Planeación y Financiación. Estudio de Costos Universitarios. Metodología. Resultados. Bogotá, 1976.

ICOLPE. Consideraciones acerca de la investigación Educativa en Colombia. Bogotá. 1974. 
ICOLPE. Centro Nacional de Documentación e Información Pedagógica. Estudios sobre Educación en Colombia. 1958-1973. Bogotá. 1975.

JALLADE, Jean Pierre. Public Expenditures on Education and Income Distribution in Colombia. Washington. International Bank of Reconstruction and Development. 1974. Rey. Plan, y Des. DNP. 8(3). 21-38, 1976.

KUGLER. Bernardo. Influencia de la Educación en los Ingresos de Trabajo: El Caso Colombiano". Revista de Planeación y Desarrollo. Bogotá, 1974.

KUGLER, Bernardo. Earnings Functions for Colombian Urban Labor Paree. University of Minessota. (mimeo). Minneapolis, 1975.

KUGLER, B. y Alejandro Vivas. Efecto del Gasto Público en la Educación sobre Variables Poblacionales en el Largo Plazo. CCRP. Área Socio-Económica. Documento Técnico $\mathrm{N}^{0} 14,1975$.

KUGLER, B. Reyes, a. y Martha I. de Gómez, Educación y Mercado de Trabajo Urbano en Colombia: Una Comparación entre Sectores Modernos y no Modernos. CCRP. Área Socio-Económica. Monografía N010. 1979.

LEBOT, Ivon. "Elementos para la Historia de la Educación en Colombia en el Siglo XX", Boletín Mensual de Estadística (DANE). 21(249)123-202, 1972.

LEBOT, Ivon. El Sistema Escolar Colombiano. Organización y Funcionamiento del Sistema Educativo Colombiano: Sistema de Producción, Escolaridad y Movilidad Social en Colombia. DANE, Bogotá, 1978.

MINISTERIO DE EDUCACION NACIONAL. Universidad Pedagógica Nacional (ICOLPE). Estudios sobre los Costos de la Educación Media Oficial en Colombia. Informe Final. Bogotá, 1973.

MINISTERIO DE EDUCACION NACIONAL. Elementos para el Estudio de la Economía y la Educación Rural en Colombia: Un Análisis de Caso. Universidad Pedagógica. Bogotá. 1976.

MINISTERIO DE EDUCACION NACIONAL. ICETEX. Selectividad del Sistema Universitario Colombiano. Papel del Crédito Educativo. Bogotá. 1976, (378-C718s). CCRP. LB 2321CO 159s.

MINISTERIO DE EDUCACION NACIONAL. Educación para el Desarrollo Rural de Colombia. Bogotá, 1978.

MINISTERIO DEL TRABAJO Y SEGURIDAD SOCIAL. Servicio Nacional de Aprendizaje (SENA). 20 Años del SENA en Colombia 1957-1977. Trabajo realizado para el SENA por Ricardo Lucia A. y la Fundación para la Educación Superior y el Desarrollo. Editorial Precia. Bogotá. 1978.

MOJICA, Guillermo. Problemas y Perspectivas de la Educación en Colombia. En Controversias sobre Economía Colombiana. U. del Externado de Col. Bogotá, 1976. 
MOJICA. Guillermo. "El Rendimiento Escolar y el Problema de Igualdad de Oportunidades". Revista Colombiana de Educación. CIUP. Universidad Pedagógica Nacional. N01. Bogotá. 1978.

PARRA, Rodrigo. Education and Dependency. "The Colombian Educational Sistem as an Agent of Undervelopment". Michigan. Xerox. 1972. University of Wisconsin. Sociology,

PARRA. Rodrigo. Dependency and Education in Colombian Undervelopement. Madison. Land Tenure Center. University of Wisconsin. 1973.

PARRA, Rodrigo. Análisis de un Mito: La Educación como Factor de Movilidad Social en Colombia. Bogotá. Universidad de los Andes. 1974.

PARRA, Rodrigo. La Educación Rural en la Zona Cafetera Colombiana. Buenos Aires. UNESCO-CEPAL-PNUD. 1978.

RAMA. Germán. Educación Universitaria y Movilidad Social: reclutamiento de élítes en Colombia.. Bogotá, Universidad Nacional, 1969.

REYES. Álvaro. Origen Socioeconómico del Estudiante Universitario. ICETEX (mimeo). 1974.

REYES, Álvaro y Patricia Restrepo. Costos Escolares de la Educación Pública en Colombia. Bogotá, CCRP, Mayo de 1979.

REYES. Álvaro y Martha Isabel de Gómez. El Papel y las Posibilidades de la Educación Técnica: El Cuso Colombiano. Bogotá. Agosto de 1979. CCRP. Área Socio-Económica.

RODRIGUEZ, F. Jaime. Las Transformaciones del Sistema Educativo y su Repercusión sobre Universidad de la Década del 80 en Colombia. Universidad Nacional de Colombia. Departamento de Sociología. (mimeo). Segunda Edición. Bogotá, 1977.

ROUX RENGIFO, José de. Metodología de investigación de la Equidad en la Inversión y Consumo de Educación Aplicado a Tres Ciudades Colombianas. Bogotá. Universidad de los Andes. 1973.

SCHULTS, Paul. Returns to Education in Bogotá-Colombia. Santa Mónica. California Rand Corporation. 1968.

SELOWSKY, Marcelo. "El Efecto del Desempleo y el Crecimiento sobre la Rentabilidad de la Inversión Educacional: Una Aplicación a Colombia". CEDE. 331:11/S46. CCRP. Revista del Departamento Nacional de Planeación. 1(2) 5-68, 1969.

SELOWSKY, Marcelo. Who Benefits from Government Expenditures? A Case Study of Colombia. World Bank. Oxford University Press. Washington. 1979.

URRUTIA, Miguel. La Educación como Factor de Movilidad Social. Bogotá. CEDE. Documento $\mathrm{N}^{0} 12,1974$.

URRUTIA, Miguel. La Distribución del ingreso y la Distribución de la Educación. El Sector Financiero y la Distribución del Ingreso. FEDESARROLLO. Bogotá, 1974. 